\title{
Balloon closure of a surgical aorto-atrial communication
}

\author{
R HAYWARD, * B KENDALL, $\dagger$ T TREASURE $\ddagger$ \\ From the Departments of *Cardiology, $†$ Imaging, and $\ddagger$ Cardiac Surgery, the Middlesex Hospital, London
}

SUMMARY Surgical repair of an extensive dissection of the proximal aorta (Shumway type A or DeBakey type I) was complicated by persistent haemorrhage from the surgical suture lines and via the false lumen. This was controlled by closing the aortic adventitia round the repaired aorta and by creating an anastomosis between the subadventitial space and the right atrial appendage. Though $\bigcup_{\infty}$ the haemorrhage was contained, the left to right (aorto-atrial) shunt led to a severe low output state, 을 which was corrected by percutaneous closure of the fistula with a detachable balloon. A year after $\rightarrow$ operation computed axial tomographic scanning showed the balloon in place though the paraaortic space persisted and communicated freely with the aorta.

\section{Case report}

A 72 year old woman was referred from another London centre. Two days before she had been admitted with a seven hour episode of severe retrosternal chest pain that culminated in collapse. There was a history of treated hypertension, cigarette smoking, and airways obstruction. She was in pain and drowsy with a systolic blood pressure of 80 $\mathrm{mm} \mathrm{Hg}$. Heart sounds were soft and pulses were normal. The initial chest radiograph showed mediastinal widening and a left pleural effusion. Serial electrocardiograms showed inverted $T$ waves and ST segment depression in the anterolateral leads. The concentration of blood urea was $8.7 \mathrm{mmol} / 1$, serum creatinine was $75 \mu \mathrm{mol} / \mathrm{l}$, haemoglobin was $10.4 \mathrm{~g} / \mathrm{dl}$, and the cell white count was $9 \cdot 2 \times 10^{9} / 1$. A computed tomographic scan showed a Shumway type A dissection of the aorta extending from the annulus of the aortic valve to the descending thoracic aorta, with a small pericardial effusion.

Because of a rapid rise in blood pressure she was treated with hypotensive agents but the aorta continued to widen. Radiographic contrast aortography showed a type $\mathbf{A}$ dissection of the aorta with an intimal tear approximately $4 \mathrm{~cm}$ above the aortic annulus, a competent aortic valve, and mid-course occlusion of a non-dominant right coronary artery. Because of these findings an operation was performed. ${ }^{12}$

Requests for reprints to Dr R Hayward, Cardiology Department, Middlesex Hospital, Mortimer Street, London WIN 8AA.
At operation, the arterial pressure was controlled with nitroprusside and the left atrial pressure was monitored indirectly. An atriofemoral bypass was established, the patient was cooled to $18 \mathrm{C}$, and thiopentone $(2 \mathrm{~g})$ was administered for cerebral protection. Total circulatory arrest lasted $35 \mathrm{~min}$ utes. When the aortic dissection sac, which affected the entire thoracic aorta, was incised an unusual longitudinal intimal entry tear was identified quite high in the ascending aorta. The ascending aorta was replaced with $24 \mathrm{~mm}$ Meadox hemishield, and the repair was wrapped in adventitia. ${ }^{2}$ The aorta distal to the repair continued to distend and a further large intimal tear in the arch slightly distal to the left subclavian origin (the probable reentry site) was located and treated by the insertion of buttressed sutures. Despite these measures profuse haemorrhage continued via the adventitia enclosing the ascending aorta. This was consistent with one or more defects of the suture line, and a fatal outcome seemed probable. As a lifesaving measure, the ơ haemorrhage was controlled by fashioning a communication between the subadventitial space and the right atrial appendage; this decompressed the paraaortic lumen by allowing blood to return to the venous circuit. Without this manoeuvre there was no prospect of weaning the patient off bypass. After $\mathbb{Q}$ construction of the anastomosis, bypass was stopped without difficulty.

Hopes that shunting from the aorta would decline because of thrombus formation in the para-aortic space were not realised. Progress over the next 48 hours was satisfactory, but thereafter the left to right 

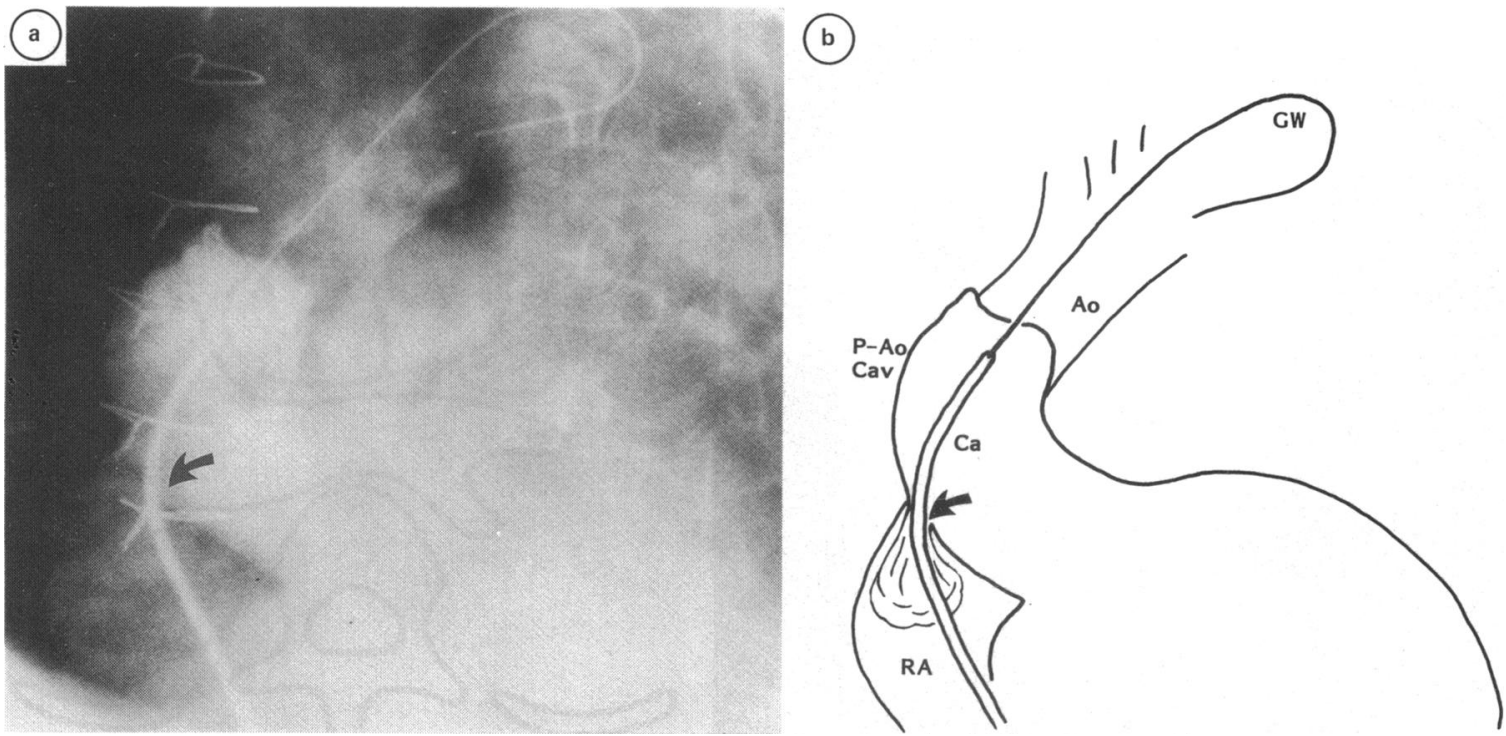

Fig 1 A single frame (left anterior oblique view) from a cineaortogram exposed during positioning of 0.38 gauge guide wire (GW) and 8.8 French gauge introducing catheter (Ca) across the aorto-atrial fistula during placement of the Debrun apparatus. Contrast was injected via the introducing catheter into the para-aortic cavity (P-Ao Cav) close to the fistula (arrow), and is seen jetting into the right atrial appendage $(R A)$. The guide wire lies in a cephalad region of the para-aortic space into which contrast has not penetrated. Contrast is principally confined to the vicinity of the prosthetic aortic replacement. Ao, aortic lumen.

(aorto-atrial) shunt became increasingly active, leading to an intractable low output state and oliguric renal failure (peak blood urea $30.5 \mathrm{mmol} / 1$ and serum creatinine $218 \mu \mathrm{mol} / \mathrm{l})$. So five weeks after the operation we used a percutaneous balloon technique to close the defect.

The right femoral vein was cannulated and a 7 French gauge Gensini catheter (Cordis UK Ltd) with a 0.38 gauge $145 \mathrm{~cm}$ long guide wire with a flexible tip (Cook UK Ltd) was advanced from the right atrium through the fistula into the para-aortic false lumen. We used a $260 \mathrm{~cm}$ exchange wire (Cook UK Ltd) to replace the Gensini catheter with an 8.8 French gauge $100 \mathrm{~cm}$ polyethylene catheter (Becton Dickinson, New Jersey, USA) (fig 1). Through this we sited a $2 \cdot 2 \mathrm{ml}$ capacity Debrun detachable balloon assembly (Ingenor Medical Systems, France (from Kimal, UK)) in the false lumen. The assembly was made up of a 2 French gauge single lumen catheter with a latex balloon attached to its tip by latex thread before use and an outer 3 French gauge coaxial catheter. The balloon was manoeuvred within the false lumen to lie close to the atrial communication. Test inflations with contrast medium (Conray 280) established how much the balloon needed to be distended to ensure that it became lodged against the fistula and prevented repassage into the right atrium. When the best site for the balloon had been established, it was drained of contrast material and refilled with the correct volume of silicone solution mixed with a chemical activator that solidified in about 20 minutes after mixing. The setting time within the balloon was determined by testing a small quantity of the mixture in a container. After the mixture had solidified the 3 French gauge outer catheter was advanced over the inner catheter to detach the balloon, which was left in position against the fistula. The introducing assembly was then withdrawn.

Before placement of the balloon the oxygen saturation of blood in both the superior and inferior venae cavae was $48 \%$ and that in the right atrium was $62 \%$. These values were unchanged immediately after the procedure, though the right atrial pressure decreased from a mean of $16 \mathrm{~mm} \mathrm{Hg}$ to $12 \mathrm{~mm} \mathrm{Hg}$. During the next 10 days, however, right ventricular overactivity decreased, heart failure cleared, and renal function returned to normal (blood urea $3.0 \mathrm{mmol} / 1$ and serum creatinine $61 \mu \mathrm{mol} / \mathrm{l})$. The patient did not agree to have further elective contrast aortography and was discharged well nine weeks after operation.

A year after operation, the aorta had not expanded further and the shunt was undetectable by clinical criteria. A computed axial tomographic scan with contrast (fig 2), however, showed that the para-aortic lumen remained open and was connected with the true lumen while the communication to the right atrium was plugged by the Debrun balloon. The patient, now aged 73 , has sustained two minor 

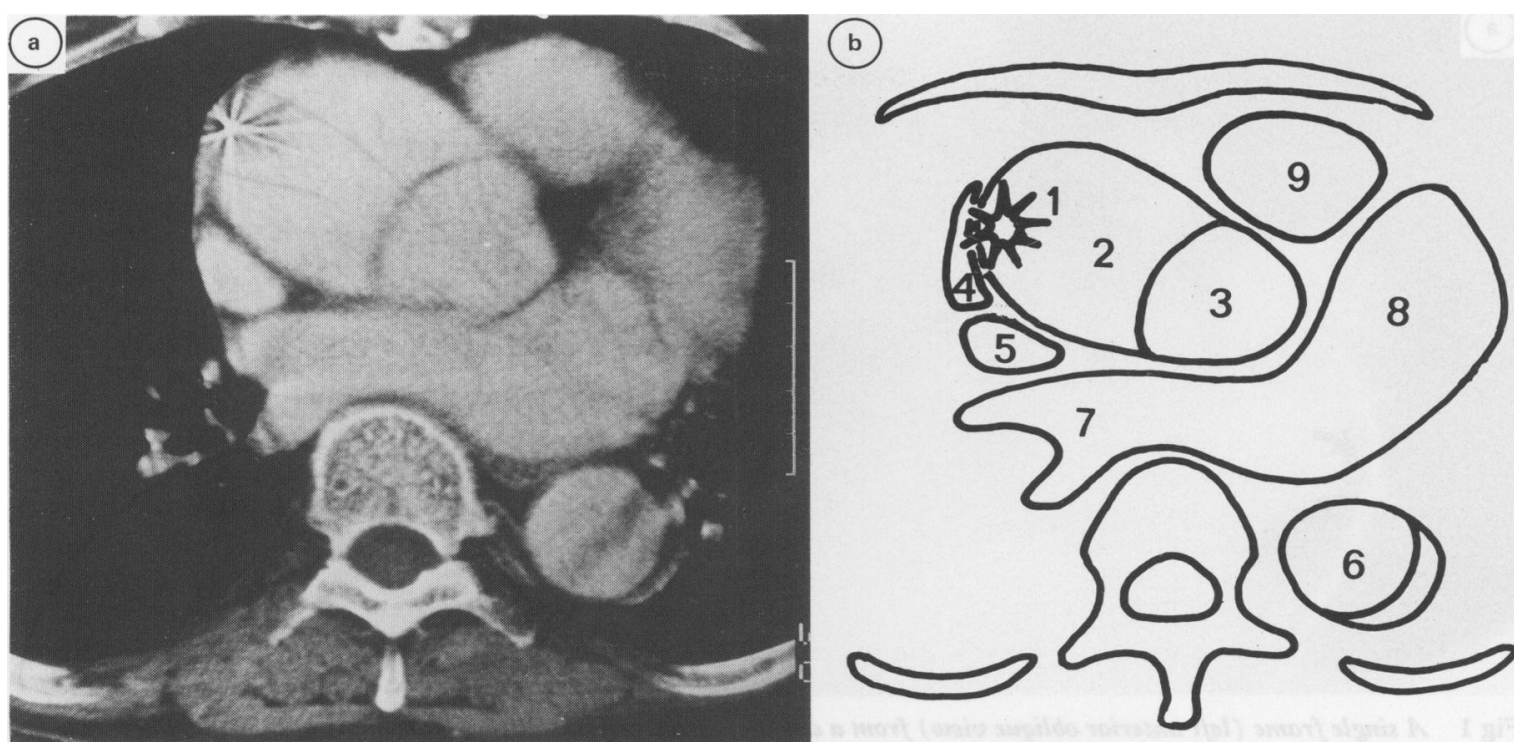

Fig 2 Computed axial tomographic scan with intravascular contrast, obtained one year after operation. Transverse cut at thơ level of the ninth thoracic vertebra showing the "starburst" effect created by the silver clip within the Debrun balloon (1), sited on the aortic aspect of the occluded aorto-atrial communication. (2) Para-aortic cavity enclosing dissected ascending aorta;

(3) true lumen of ascending aorta; (4) right atrial appendage; (5) superior vena cava; (6) descending aorta (also dissected);

(7) pulmonary veins entering left atrium; (8) left ventricle; (9) right ventricular outflow tract.

cerebrovascular accidents, and is slightly disabled. No further intervention is planned.

\section{Discussion}

This patient needed an operation to stop the expansion of a type A dissection of the aorta. ${ }^{12}$ We hoped that the communication that we created to deal with life threatening haemorrhage at operation would close spontaneously, but flow through the shunt progressed to imperil survival and make a further operation too hazardous. At first the balloon did not completely obstruct the aorto-atrial shunting, but subsequently the latex surface induced formation of thrombus, which produced more complete closure. Also the balloon may have impacted more fully with time. The Debrun apparatus is designed primarily to close fistulas and aneurysms of the large cerebral arteries. ${ }^{3}$ These carry high flows and are not end arteries. The risk of embolism of the occluding device is high in an aortic dissection and so it must be carefully positioned. The siting of the balloon is precise and potentially reversible until the final stage, unless gelfoam or steel coils are used. Detachable balloons have been used to close Blalock-Taussig shunts and congenital coronary arterioarterial and coronary arteriovenous communications. ${ }^{4} \mathrm{~A}$ double lumen intra-aortic balloon was used to close the intimal entry tear in Shumway type B dissections. ${ }^{5}$
In the present case, the risk of the balloon causingo an embolism into the aorta was accepted because of the deleterious effects of the persisting fistula. Theo surgical mortality for repair of a type $A$ aortic dissection is $15-20 \%^{1}$; haemorrhage from sutures lines in friable aortic tissues contributes signifi- $\overrightarrow{0}$ cantly. ${ }^{2}$ Decompression of the para-aortic lumen into-3 the right atrium can be a lifesaving procedure; a method by which the resulting fistula can be closeक्ष. later without surgery will be valuable.

\section{References}

1 DeSanctis RW, Doraghazi RM, Austen WG, Buckley MJ. Aortic dissection. N Engl J Med 1987;3170 1060-7.

2 Treasure T. Dissection of the thoracic aorta. In: Rusself RCG, ed. Recent advances in surgery-12. Edinburgh‥ Churchill Livingstone, 1986:231-48.

3 Debrun G, Fox A, Drake C, Peerless S, Girvin o Ferguson G. Giant unclippable aneurysms. Treat ment with detachable balloons. AJNR 1981;2 స్ 167-73.

4 Reidy JF, Jones ODH, Tynan MJ, Baker EJ, Joseph MC. Embolisation procedures in congenital heart disease. Br Heart J 1985;54:184-92.

5 Akaba N, Ujiie H, Umezawa $\mathrm{K}$, et al. Management of ${ }^{+}$ acute aortic dissections with a cylinder-type balloon catheter to close the entry. J V asc Surg 1986;3:890-4 